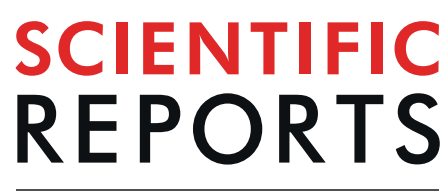

natureresearch

\title{
OPEN Carcinogenic risk of human papillomavirus (HPV) genotypes and potential effects of HPV vaccines in Korea
}

Received: 11 March 2019

Accepted: 13 August 2019

Published online: 29 August 2019

\begin{abstract}
Eunhyang Park ${ }^{1,2}$, Ji-Ye Kim ${ }^{3}$, Sangjoon Choi ${ }^{1}$, Dae Shick Kim ${ }^{1}$ \& Young Lyun Oh ${ }^{1}$
This study investigated the distribution of HPV types in Korean women and evaluated the carcinogenic risk of individual HPV types and the potential effects of HPV vaccines. A total of 4,081 HPV-positive samples between 2014 and 2017 were included. The most prevalent genotypes were HPV 16, 58, 68, and 56. Among them, HPV 16 was significantly higher in high-grade squamous intraepithelial neoplasia or worse (HSIL+) group. In cytologically evaluating the risk for HSIL + by individual HPV types, HPV 16 was associated with the highest risk of $\mathrm{HSIL}+(\mathrm{OR}=10.82 ; 95 \% \mathrm{Cl}: 7.93-14.77)$, followed by HPV $33,31,52,18,58,51$, and 35 , in descending order ( $O R=3.50$ [type 33] to 2.62 [type 35]). Among those types, HPV 16, 18, 31, 33, and 58 were also significantly associated with HSIL+ on histologic evaluation. The analysis of the HPV subgroups covered by the different vaccines revealed that the HPV types covered by the 9-valent vaccine had a high association with $\mathrm{HSIL}+(\mathrm{OR}=4.09 ; 95 \% \mathrm{Cl}: 3.02-5.54)$. Our findings highlight the different carcinogenic risks posed by the high risk HPV genotypes and the positive potential effects of the 9-valent HPV vaccine in reducing HPV-associated cervical cancer in Korea.
\end{abstract}

Human papillomaviruses (HPVs) are a well-known cause of cervical intraepithelial neoplasia (CIN) and invasive cervical cancer ${ }^{1}$. Of the more than 200 identified HPV types $^{2}$, approximately 40 infect the genital area ${ }^{3}$. Of those, 13-15 high risk (HR) HPV types are known to be carcinogenic ${ }^{4}$. According to worldwide data, HPV 16 and 18 account for more than $70 \%$ of cervical cancers, and six other types (HPV 31, 33, 35, 45, 52, and 58) account for an additional $20 \%{ }^{5-7}$. However, the contribution of specific HPV types to cervical cancer differs by region, possibly because of geographic variations in HPV type-specific prevalence in different populations ${ }^{8,9}$. For example, the prevalence of HPV 16 and 18 is relatively low in East Asia in both cervical cancer patients and healthy women compared to the worldwide prevalence $e^{5,8,10}$. Therefore, it is important to understand the prevalence of specific HPV types on a population-specific basis when developing strategies to prevent cervical cancer.

Three types of vaccines are currently available to prevent cervical cancer: 2-valent (Cervarix, GlaxoSmithKline, Rixensart, Belgium), 4-valent (Gardasil, Merck, Whitehouse Station, NJ), and 9-valent (Gardasil 9, Merck, Whitehouse Station, NJ) vaccines. The 2- and 4-valent vaccines protect against HPV 16 and 18, which are the two major carcinogenic types, and the 9-valent vaccine further protects against HPV 31, 33, 45, 52, and 58. Although these vaccines are expected to address approximately $70 \%$ and $90 \%$ of cervical cancers, respectively ${ }^{5,6}$, their preventive effects need to be evaluated on a regional basis.

The risk that an HPV infection will progress to cervical cancer varies according to the HPV genotype ${ }^{4,7}$. The International Agency for Research on Cancer (IARC) Working Group defined HPV types as carcinogenic if their prevalence in cancers was significantly greater than that of HPV type 6, the archetype low risk (LR) HPV type, and if they were significantly more common in cancer patients than in the general population ${ }^{11}$. Although a number of studies have reported the distribution of HPV types in cervical cancer patients or healthy women, few studies have evaluated the carcinogenic potential of individual HPV genotypes by analyzing case-controlled differences, as in the IARC definition ${ }^{12-14}$. Moreover, case-control studies rarely provide statistically valid results

${ }^{1}$ Department of Pathology and Translational Genomics, Samsung Medical Center, Sungkyunkwan University School of Medicine, Seoul, Korea. '2Department of Pathology, Yonsei University College of Medicine, Seoul, Korea. ${ }^{3}$ Department of Pathology, Ilsan Paik Hospital, Inje University, Goyang, Korea. Correspondence and requests for materials should be addressed toY.L.O. (email: bijou@skku.edu) 


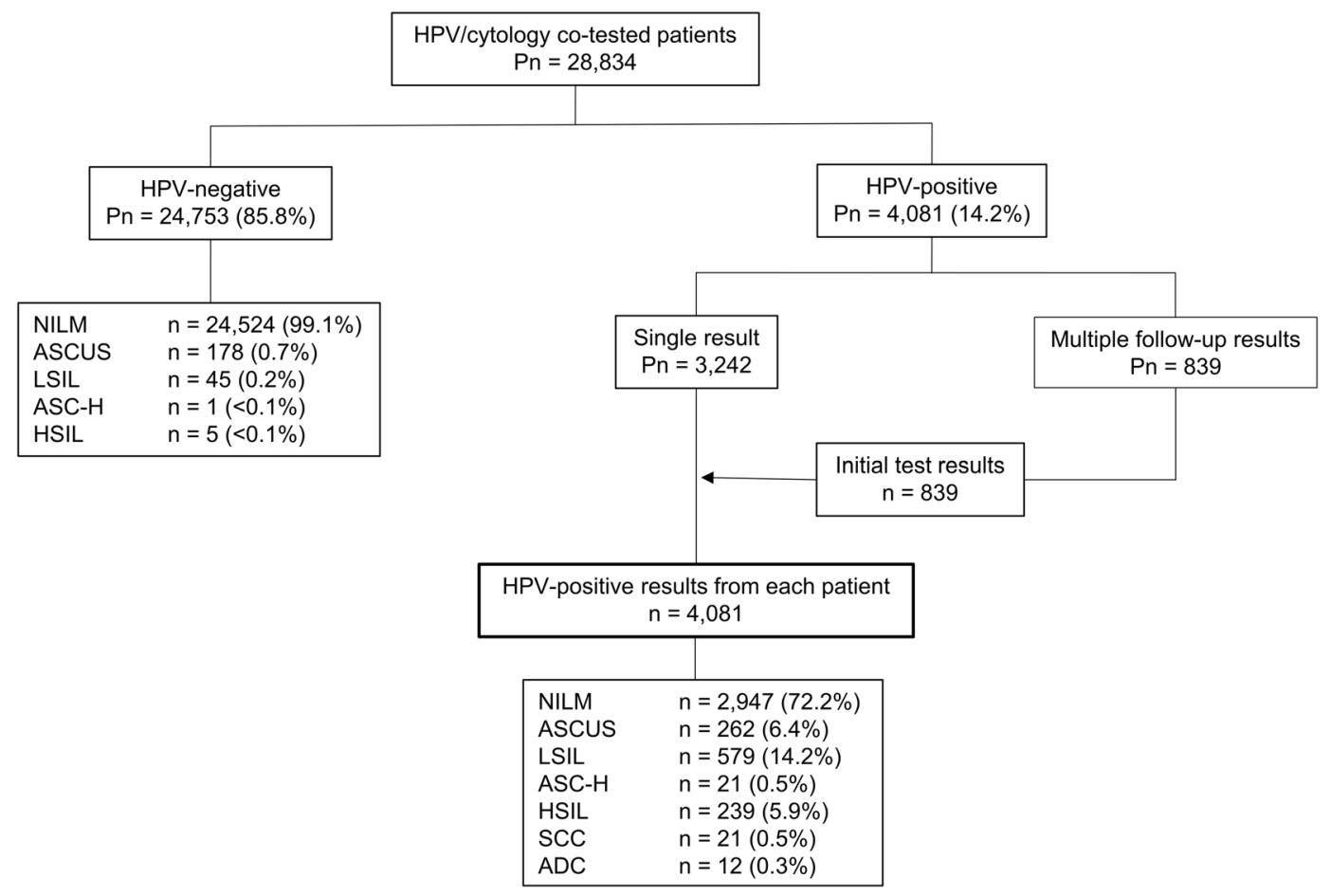

Figure 1. Flow diagram of patient selection. $\mathrm{ADC}=$ adenocarcinoma, $\mathrm{ASC}-\mathrm{H}=$ atypical squamous cells-cannot exclude high-grade squamous intraepithelial neoplasia, ASCUS = atypical squamous cells of undetermined significance, HSIL = high-grade squamous intraepithelial neoplasia, LSIL = low-grade squamous intraepithelial neoplasia, NILM = negative for intraepithelial lesions or malignancy, $\mathrm{Pn}=$ number of patients, $\mathrm{SCC}=$ squamous cell carcinoma.

because of the low prevalence of HPV infection in the cohort population. Therefore, it could be beneficial to estimate the association between individual HPV types and cervical cancer in an HPV-positive group. In this study, we evaluated the distribution of HPV types in Korean women and estimated the carcinogenic potential of individual HPV types by considering the association between specific HPV types and cervical cancer or precancerous lesions. In addition, we evaluated the potential effects of HPV vaccines in Korea.

\section{Results}

Study population and sample evaluation. Among women who underwent HPV genotyping at Samsung Medical Center between October 2014 and March 2017, we retrieved the anonymized records of patients with co-tested cervical cytology and HPV genotyping results. For the cytology specimens, Papanicolaou-stained liquid-based tests were performed with using a ThinPrep 5000 Processor (Hologic Inc., Malborough, MA) according to the manufacturer's protocol. Pathologists diagnosed the cytology slides according to the 2001 Bethesda system for cervicovaginal cytology: negative for intraepithelial lesions or malignancy (NILM); atypical squamous cells of undetermined significance (ASCUS); low-grade squamous intraepithelial neoplasia (LSIL); atypical squamous cells-cannot exclude high-grade squamous intraepithelial neoplasia (ASC-H); high-grade squamous intraepithelial neoplasia (HSIL); squamous cell carcinoma (SCC); and adenocarcinoma (ADC) ${ }^{15}$. For our analysis, we used three subgroups based on the cytologic diagnoses: NILM, LSIL (including ASCUS and LSIL), and HSIL+ (including ASC-H, HSIL, SCC, and ADC).

Of the 28,834 women with cervical cytology and HPV results, $91.5 \%$ received HPV genotyping as part of a routine health check-up, and $8.5 \%$ were referred from clinics due to abnormal cervical cytology results or other gynecologic problems. Among the 24,753 (85.8\%) HPV-negative women, $0.9 \%$ had abnormal cytology results, including ASCUS (0.7\%), LSIL (0.2\%), ASC-H $(<0.1 \%)$, and HSIL $(<0.1 \%)$. Among the 4,081 HPV-positive patients, 3,242 patients had a single HPV result with a matched cytology result, and 839 patients had one or more follow-up results. We analyzed 4,081 (14.2\%) HPV-positive results, including both single and initial results (Fig. 1). Among the HPV-positive patients, 596 patients (14.6\%) underwent a follow-up biopsy within a year of the initial cervical pap test. The follow-up colposcopic biopsy specimens were stained with hematoxylin and eosin and diagnosed as NILM, LSIL, HSIL, SCC, or ADC. For our statistical analyses, the histologic diagnoses were also classified as NILM, LSIL, or HSIL+ (including HSIL, SCC, and ADC).

Characteristics of the study population. The median age of the HPV-positive patients was 52 years (range: 17 to 86 years). Cytologic diagnoses were NILM in $72.2 \%$, ASCUS in 6.4\%, LSIL in 14.2\%, ASC-H in 0.5\%, HSIL in $5.9 \%$, SCC in $0.5 \%$, and ADC in $0.3 \%$. The most common HPV type was HPV 16 (8.7\%), followed by 


\begin{tabular}{|c|c|c|c|c|c|}
\hline & \multicolumn{4}{|l|}{ n (\%) } & \multirow[b]{2}{*}{ p-value } \\
\hline & All & NILM & LSIL & HSIL+ & \\
\hline All & 4081 & $2947(72.2)$ & $841(20.6)$ & $293(7.2)$ & \\
\hline \multicolumn{6}{|l|}{ Age (years) } \\
\hline Median (Q1-Q3) & $52(45-58)$ & $53(47-59)$ & $49(40-55)$ & $45(36-54)$ & $<0.001^{\mathrm{a}}$ \\
\hline$\leq 34$ & $362(8.9)$ & $174(5.9)$ & $131(15.6)$ & $57(19.5)$ & $<0.001^{\mathrm{b}, \mathrm{c}, \mathrm{d}}$ \\
\hline $35-44$ & $588(14.4)$ & $348(11.8)$ & $151(18.0)$ & $89(30.4)$ & \\
\hline $45-54$ & $1528(37.4)$ & 1127 (38.2) & $321(38.2)$ & $80(27.3)$ & \\
\hline $55-64$ & $1261(30.9)$ & $1025(34.8)$ & $192(22.8)$ & $44(15.0)$ & \\
\hline$\geq 65$ & \begin{tabular}{|l|}
$342(8.4)$ \\
\end{tabular} & $273(9.3)$ & $46(5.5)$ & $23(7.8)$ & \\
\hline \multicolumn{6}{|l|}{ HR HPV infection } \\
\hline Yes & 2400 (58.8) & $1565(53.1)$ & $555(66.0)$ & $280(95.6)$ & $<0.001$ \\
\hline No & $1681(41.2)$ & $1382(46.9)$ & $286(34.0)$ & $13(4.4)$ & \\
\hline \multicolumn{6}{|l|}{ HR HPV genotypes } \\
\hline 16 & $356(8.7)$ & $186(6.3)$ & $64(7.6)$ & $106(36.2)$ & $<0.001^{\mathrm{c,d}}$ \\
\hline 18 & $140(3.4)$ & $96(3.3)$ & $24(2.9)$ & $20(6.8)$ & $0.004^{\mathrm{c}, \mathrm{d}}$ \\
\hline 31 & $113(2.8)$ & $75(2.5)$ & $21(2.5)$ & $17(5.8)$ & $0.005^{c, d}$ \\
\hline 33 & $128(3.1)$ & $71(2.4)$ & $37(4.4)$ & $20(6.8)$ & $<0.001^{\mathrm{b}, \mathrm{d}}$ \\
\hline 35 & $133(3.3)$ & $79(2.7)$ & $37(4.4)$ & $17(5.8)$ & $0.002^{\mathrm{d}}$ \\
\hline 39 & $247(6.1)$ & $177(6.0)$ & $62(7.4)$ & $8(2.7)$ & $0.016^{c}$ \\
\hline 45 & $62(1.5)$ & $45(1.5)$ & $16(1.9)$ & $1(0.3)$ & 0.17 \\
\hline 51 & $229(5.6)$ & $130(4.4)$ & $73(8.7)$ & $26(8.9)$ & $<0.001^{\mathrm{b}, \mathrm{d}}$ \\
\hline 52 & $220(5.4)$ & $126(4.3)$ & $65(7.7)$ & $29(9.9)$ & $<0.001^{\mathrm{b}, \mathrm{d}}$ \\
\hline 56 & \begin{tabular}{|l|}
$281(6.9)$ \\
\end{tabular} & $169(5.7)$ & $95(11.3)$ & $17(5.8)$ & $<0.001^{\mathrm{b}, \mathrm{c}}$ \\
\hline 58 & $318(7.8)$ & $216(7.3)$ & $64(7.6)$ & $38(13.0)$ & $0.003^{c, d}$ \\
\hline 59 & $43(1.1)$ & $26(0.9)$ & $12(1.4)$ & $5(1.7)$ & 0.21 \\
\hline 66 & $159(3.9)$ & $98(3.3)$ & $49(5.8)$ & $12(4.1)$ & $0.004^{\mathrm{b}}$ \\
\hline 68 & $309(7.6)$ & $228(7.7)$ & $63(7.5)$ & $18(6.1)$ & 0.61 \\
\hline \multicolumn{6}{|c|}{ Single vs. multiple HR HPV infection } \\
\hline Single & 2117 (51.9) & $1425(48.4)$ & $458(54.5)$ & $234(79.9)$ & $<0.001^{\mathrm{b}, \mathrm{d}}$ \\
\hline Multiple & $283(6.9)$ & $140(4.8)$ & $97(11.5)$ & $46(15.7)$ & \\
\hline \multicolumn{6}{|l|}{ Vaccine subgroups } \\
\hline $16 / 18$ & $488(12.0)$ & $277(9.4)$ & $88(10.5)$ & $123(42.0)$ & $<0.001^{c, d}$ \\
\hline $31 / 33 / 45 / 52 / 58$ & $807(19.8)$ & $518(17.6)$ & $187(22.2)$ & $102(34.8)$ & $<0.001^{\mathrm{b}, c, \mathrm{~d}}$ \\
\hline $35 / 39 / 51 / 56 / 59 / 66 / 68$ & $1293(31.7)$ & $867(29.4)$ & $338(40.2)$ & $88(30.0)$ & $<0.001^{\mathrm{b}, \mathrm{c}}$ \\
\hline \multicolumn{6}{|l|}{ LR HPV genotypes } \\
\hline 6 & $96(2.4)$ & $64(2.2)$ & $27(3.2)$ & $5(1.7)$ & 0.16 \\
\hline 11 & $83(2.0)$ & $52(1.8)$ & $30(3.6)$ & $1(0.3)$ & 0.001 \\
\hline 34 & $74(1.8)$ & $52(1.8)$ & $20(2.4)$ & $2(0.7)$ & 0.16 \\
\hline 40 & $185(4.5)$ & $135(4.6)$ & $43(5.1)$ & $7(2.4)$ & 0.15 \\
\hline 42 & \begin{tabular}{|l|}
$196(4.8)$ \\
\end{tabular} & $135(4.6)$ & $57(6.8)$ & $4(1.4)$ & 0.001 \\
\hline Others & $1238(30.3)$ & $1035(35.1)$ & $194(23.1)$ & $9(3.1)$ & $<0.001$ \\
\hline
\end{tabular}

Table 1. The distribution of patient ages and HPV genotypes according to cytologic diagnoses. ${ }^{\text {aSignificant }}$ differences in Dunn's test $(\alpha=0.05)$ were found between the NILM and LSIL groups, the LSIL and HSIL+ groups, and the NILM and HSIL+ groups. ${ }^{\mathrm{b}} \mathrm{A}$ significant p-value after Bonferroni correction $(<0.0167)$ was found between the NILM and LSIL groups. ${ }^{c}$ A significant p-value after Bonferroni correction $(<0.0167)$ was found between the LSIL and HSIL+ groups. ${ }^{\mathrm{d}} \mathrm{A}$ significant $\mathrm{p}$-value after Bonferroni correction $(<0.0167)$ was found between the NILM and HSIL+ groups. HR = high risk, HSIL+ = high-grade squamous intraepithelial neoplasia or worse, $\mathrm{LR}=$ low risk, $\mathrm{LSIL}=$ low-grade squamous intraepithelial neoplasia, NILM = negative for intraepithelial lesions or malignancy.

HPV 58 (7.8\%), HPV 68 (7.6\%), and HPV 56 (6.9\%). The distributions of patient ages and HPV genotypes are summarized in Table 1.

Of all HPV-tested women, 5,262 (18.2\%) received one or more follow-up tests. Of them, 3,745 women were HPV-negative and 1,517 patients were HPV-positive in their initial tests. Among initially HPV-positive patients, 839 patients (55.3\%) were also HPV positive in their follow-up tests, whereas HPV infection of the remaining 678 patients (44.7\%) had been cleared. In addition, cytologic subgroups of 209 patients (16.7\%) had changed in their follow-up results (Supplementary Table S1). 


\begin{tabular}{|c|c|c|}
\hline Variables & OR $(95 \% \mathrm{CI})$ & p-value \\
\hline \multicolumn{3}{|c|}{ Age group (years) } \\
\hline$\leq 34$ & 1 & \\
\hline $35-44$ & $1.08(0.72-1.61)$ & 0.017 \\
\hline $45-54$ & $0.36(0.24-0.53)$ & $<0.001$ \\
\hline $55-64$ & $0.23(0.15-0.36)$ & 0.002 \\
\hline$\geq 65$ & $0.42(0.24-0.73)$ & $<0.001$ \\
\hline \multicolumn{3}{|c|}{ HR HPV types } \\
\hline 16 & $11.75(8.55-16.15)$ & $<0.001$ \\
\hline 18 & $3.26(1.85-5.76)$ & $<0.001$ \\
\hline 31 & $3.38(1.85-6.18)$ & $<0.001$ \\
\hline 33 & $3.50(2.00-6.12)$ & $<0.001$ \\
\hline 35 & $2.62(1.46-4.72)$ & 0.001 \\
\hline 39 & $0.50(0.23-1.11)$ & 0.090 \\
\hline 45 & $0.40(0.05-2.95)$ & 0.367 \\
\hline 51 & $2.74(1.71-4.37)$ & $<0.001$ \\
\hline 52 & $3.34(2.10-5.33)$ & $<0.001$ \\
\hline 56 & $1.49(0.87-2.58)$ & 0.149 \\
\hline 58 & $2.83(1.88-4.28)$ & $<0.001$ \\
\hline 59 & $1.79(0.64-5.01)$ & 0.268 \\
\hline 66 & $1.61(0.84-3.08)$ & 0.151 \\
\hline 68 & $1.56(0.92-2.64)$ & 0.102 \\
\hline
\end{tabular}

Table 2. Multivariate logistic regression analyses of age groups and HR HPV types according to HSIL+ versus NILM/LSIL in the cytology diagnoses. $\mathrm{CI}=$ confidence interval, $\mathrm{HR}=$ high risk, HSIL $+=$ highgrade squamous intraepithelial neoplasia or worse, LSIL = low-grade squamous intraepithelial neoplasia, $\mathrm{NILM}=$ negative for intraepithelial lesions or malignancy, $\mathrm{OR}=$ odds ratio.

Distribution of HPV types according to cytologic subgroups. The NILM cytology group showed a high prevalence of HPV 68 (7.7\%), HPV 58 (7.3\%), and HPV 16 (6.3\%). In the LSIL group, HPV 56 was the most common HPV type (11.3\%), followed by HPV 51 (8.7\%), HPV 52 (7.7\%), and HPV 58 and 16 (7.6\%). In the HSIL+ group, HPV $16(36.2 \%)$ was most frequently identified, followed by HPV 58 and $52(13.0 \%$ and $9.9 \%$, respectively). The proportion of HR HPV infections increased significantly moving through the NILM, LSIL, and HSIL+ groups, with percentages of $53.1 \%, 66.0 \%$, and $95.6 \%$, respectively (p-value $<0.05$ ). Among the HR HPV types, HPV 16, 18, 31, 33, 35, 39, 51, 52, 56, 58, and 66 were differently distributed in the cytologic subgroups (Bonferroni's corrected p-values $<0.0167$ ) (Table 1).

Carcinogenic risk of individual HR HPV types. To assess the association of individual HPV types with HSIL+, we estimated the odds ratio (OR) of having HSIL+ (compared to NILM/LSIL) according to the individual HPV types. After adjusting for age and all other HPV types, HPV 16 was associated with the highest risk of HSIL $+(\mathrm{OR}=11.75 ; 95 \% \mathrm{CI}$ : 8.55-16.15). In addition, HPV 33, 31, 52, 18, 58, 51, and 35, in descending order, were significantly associated with an increased risk of HSIL+ (OR ranged from 3.50 [HPV 33] to 2.62 [HPV 35]). Those data are summarized in Table 2 and visualized in Fig. 2.

Among HPV-positive patients, 596 underwent a follow-up biopsy according to the "Practice guidelines for the early detection of cervical cancer in Korea" ${ }^{16}: 0.7 \%$ of NILM patients, $3.1 \%$ of ASCUS patients, $55.3 \%$ of LSIL patients, $4.8 \%$ of ASC-H patients, $89.5 \%$ of HSIL patients, and $100 \%$ of SCC and ADC patients. Among patients with HSIL + cytology, $84.3 \%$ were histologically confirmed as HSIL +. The correlations between histology and cytology diagnoses is shown in Table 3. We evaluated the association between individual HPV types and a histology-based HSIL + result. After adjusting for age and HPV types, HPV type 16 was the most strongly associated with HSIL + $(\mathrm{OR}=9.22$; 95\% CI: 5.46-15.56), followed by HPV 31, 59, 58, 18, and 33, in descending order (OR ranged from 6.99 [HPV 31] to 3.28 [HPV 33]). Those data are shown in Table 4 and Supplementary Fig. S1.

Potential effects of HPV vaccines. We further analyzed the HR HPV types according to vaccine coverage. HPV 16 and 18, which are covered by all HPV vaccines, were present in $12.0 \%$ of our HPV-positive patients (Table 1). HPV 31, 33, 45, 52, and 58, which are covered by the 9-valent vaccine, accounted for $19.8 \%$ of HPV-positive patients in our population. The remaining HR types (HPV 35, 39, 51, 56, 59, 66, and 68) accounted for $31.7 \%$ of our HPV-positive patients. In the HSIL+ group, the proportions of HPV 16/18 and HPV $31 / 33 / 45 / 52 / 58$ were $42.0 \%$ and $34.8 \%$, respectively. Our analysis of the cytologic results revealed that HPV 16/18 showed the highest risk for HSIL+, with an OR of 10.82 (95\% CI: 7.93-14.77), followed by HPV 31/33/45/52/58 and 35/39/51/56/59/66/68, with ORs of 4.09 (95\% CI: 3.02-5.54) and 1.92 (95\% CI: $1.41-2.60$ ), respectively (Table 5). Our analysis of the histologic results revealed that the ORs of HPV 16/18, HPV 31/33/45/52/58, and HPV 35/39/51/56/59/66/68 for HSIL+ were 9.46 (95\% CI: 5.60-15.97), 3.93 (95\% CI: 2.48-6.22), and 1.58 (95\% CI: 1.00-2.49), respectively (Table 6). 


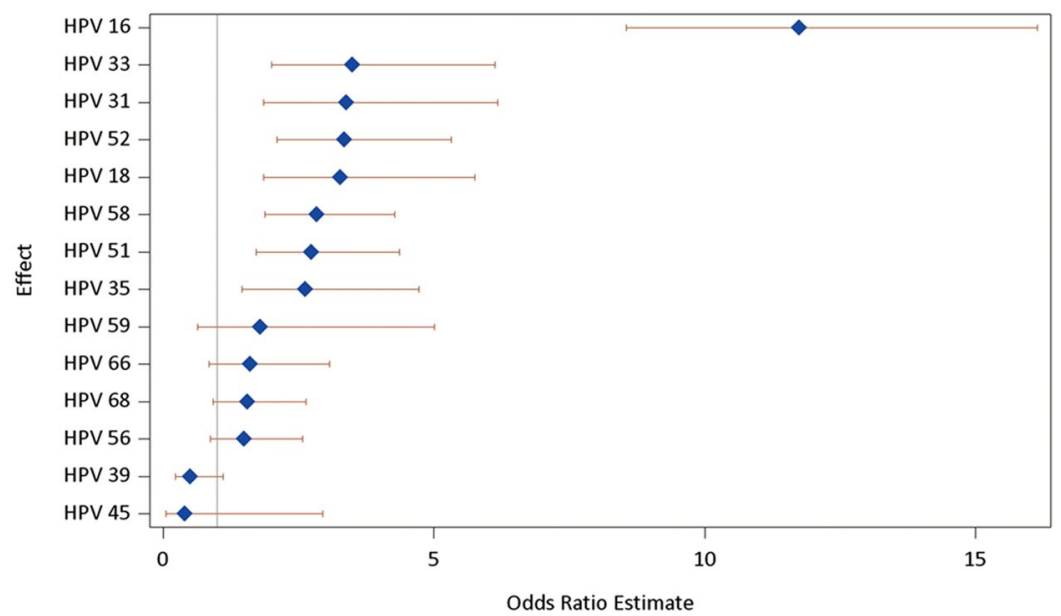

Figure 2. Logistic regression analyses of HPV genotypes based on cytologic results.

\begin{tabular}{|l|l|l|l|l|l|l|l|}
\hline $\begin{array}{l}\text { Histologic } \\
\text { diagnosis }\end{array}$ & $\begin{array}{l}\text { NILM } \\
(\mathbf{n}=\mathbf{2})\end{array}$ & $\begin{array}{l}\text { LSIL } \\
(\mathbf{n}=\mathbf{3 4 4})\end{array}$ & $\begin{array}{l}\text { HSIL } \\
(\mathbf{n}=\mathbf{2 1 3})\end{array}$ & $\begin{array}{l}\text { SCC } \\
(\mathbf{n = 2 5})\end{array}$ & $\begin{array}{l}\text { ADC } \\
(\mathbf{n = 1 2})\end{array}$ & $\begin{array}{l}\text { Total } \\
(\mathbf{n}=\mathbf{5 9 6})\end{array}$ \\
\hline Cytology \\
\hline NILM & $2(100.0 \%)$ & $18(5.2 \%)$ & $0(0.0 \%)$ & $0(0.0 \%)$ & $0(0.0 \%)$ & 20 \\
\hline ASCUS & $0(0.0 \%)$ & $7(2.0 \%)$ & $1(0.5 \%)$ & $0(0.0 \%)$ & $0(0.0 \%)$ & 8 \\
\hline LSIL & $0(0.0 \%)$ & $316(91.9 \%)$ & $4(1.9 \%)$ & $0(0.0 \%)$ & $0(0.0 \%)$ & 320 \\
\hline ASC-H & $0(0.0 \%)$ & $1(0.3 \%)$ & $0(0.0 \%)$ & $0(0.0 \%)$ & $0(0.0 \%)$ & 1 \\
\hline HSIL & $0(0.0 \%)$ & $2(0.6 \%)$ & $207(97.2 \%)$ & $5(20.0 \%)$ & $0(0.0 \%)$ & 214 \\
\hline SCC & $0(0.0 \%)$ & $0(0.0 \%)$ & $1(0.5 \%)$ & $20(80.0 \%)$ & $0(0.0 \%)$ & 21 \\
\hline ADC & $0(0.0 \%)$ & $0(0.0 \%)$ & $0(0.0 \%)$ & $0(0.0 \%)$ & $12(100.0 \%)$ & 12 \\
\hline
\end{tabular}

Table 3. Correlations between cervical histology and cytology diagnoses. ADC = adenocarcinoma, ASC-H = atypical squamous cells-cannot exclude high-grade squamous intraepithelial neoplasia, ASCUS $=$ atypical squamous cells of undetermined significance, HSIL $=$ high-grade squamous intraepithelial neoplasia, LSIL = low-grade squamous intraepithelial neoplasia, NILM = negative for intraepithelial lesions or malignancy, SCC $=$ squamous cell carcinoma.

\begin{tabular}{|l|l|c|}
\hline HPV type & OR $(\mathbf{9 5} \% \mathbf{C I})$ & p-value \\
\hline 16 & $9.22(5.46-15.56)$ & $<0.001$ \\
\hline 18 & $3.73(1.51-9.20)$ & 0.004 \\
\hline 31 & $6.99(2.85-17.13)$ & $<0.001$ \\
\hline 33 & $3.28(1.54-6.96)$ & 0.002 \\
\hline 35 & $2.24(0.94-5.33)$ & 0.070 \\
\hline 39 & $0.66(0.28-1.58)$ & 0.354 \\
\hline 45 & $0.33(0.04-2.64)$ & 0.296 \\
\hline 51 & $1.29(0.68-2.43)$ & 0.432 \\
\hline 52 & $1.67(0.85-3.25)$ & 0.134 \\
\hline 56 & $1.11(0.57-2.16)$ & 0.765 \\
\hline 58 & $3.88(2.12-7.10)$ & $<0.001$ \\
\hline 59 & $6.59(1.15-37.85)$ & 0.035 \\
\hline 66 & $1.03(0.47-2.27)$ & 0.936 \\
\hline 68 & $1.29(0.65-2.55)$ & 0.461 \\
\hline
\end{tabular}

Table 4. Multivariate logistic regression analyses of HR HPV types according to HSIL+ versus NILM/LSIL in the histologic results, adjusted for age. $\mathrm{CI}=$ confidence interval, HSIL $+=$ high-grade squamous intraepithelial neoplasia or worse, LSIL = low-grade squamous intraepithelial neoplasia, NILM= negative for intraepithelial lesions or malignancy, $\mathrm{OR}=$ odds ratio.

Age-specific HPV prevalence and its association with cytologic diagnoses. Among all of the women in our study cohort, the overall HPV prevalence was highest $(28.4 \%)$ in the $\leq 34$ years age group, and 


\begin{tabular}{|l|c|l|}
\hline Vaccine subgroup & OR $(\mathbf{9 5} \% \mathbf{C I})$ & p-value \\
\hline $16 / 18$ & $10.82(7.93-14.77)$ & $<0.001$ \\
\hline $31 / 33 / 45 / 52 / 58$ & $4.09(3.02-5.54)$ & $<0.001$ \\
\hline $35 / 39 / 51 / 56 / 59 / 66 / 68$ & $1.92(1.41-2.60)$ & $<0.001$ \\
\hline
\end{tabular}

Table 5. Multivariate logistic regression analyses of HR HPV vaccine subgroups according to HSIL+ versus NILM/LSIL in the cytology diagnoses, adjusted for age. CI = confidence interval, HR = high risk, HSIL $+=$ high-grade squamous intraepithelial neoplasia or worse, LSIL = low-grade squamous intraepithelial neoplasia, NILM = negative for intraepithelial lesions or malignancy, $\mathrm{OR}=$ odds ratio.

\begin{tabular}{|l|l|c|}
\hline Vaccine subgroup & OR $(\mathbf{9 5} \% \mathbf{C I})$ & p-value \\
\hline $16 / 18$ & $9.46(5.60-15.97)$ & 0.001 \\
\hline $31 / 33 / 45 / 52 / 58$ & $3.93(2.48-6.22)$ & $<0.001$ \\
\hline $35 / 39 / 51 / 56 / 59 / 66 / 68$ & $1.58(1.00-2.49)$ & $<0.001$ \\
\hline
\end{tabular}

Table 6. Multivariate logistic regression analyses of HR HPV vaccine subgroups according to HSIL+ versus NILM/LSIL in the histologic results, adjusted for age. $\mathrm{CI}=$ confidence interval, $\mathrm{HR}=$ high risk, HSIL $+=$ highgrade squamous intraepithelial neoplasia or worse, LSIL = low-grade squamous intraepithelial neoplasia, $\mathrm{NILM}=$ negative for intraepithelial lesions or malignancy, $\mathrm{OR}=$ odds ratio.

the prevalence tended to decrease steadily with age (Supplementary Table S2 and Fig. S2). We found no discrete trends in type-specific HPV prevalence according to age. In the association between age and cytologic diagnosis, the median age of LSIL patients was 49 years, and the prevalence of LSIL showed a single peak in the 45-54 years age group. Among HSIL+ patients, the median age was 45 years, with a peak at 35-44 years followed by a decrease with age. The ages of the NILM, LSIL, and HSIL+ cytologic groups differed significantly (Bonferroni's corrected $p$-value $<0.0167)$. Our logistic regression analyses showed that older patients ( $>45$ years) had an inverse risk for HSIL+ (Table 2).

Infection by multiple HR HPV genotypes. HR HPV types were co-infected in $6.9 \%$ of HPV-positive patients, with two to six concomitant HPV types (Supplementary Table S3). The percentage of multiple infections increased significantly from $4.8 \%$ in the NILM cytologic subgroup to $11.5 \%$ in the LSIL subgroup and $15.7 \%$ in the HSIL + subgroup ( $\mathrm{p}<0.05)$. HPV $58(24.7 \%), 16(21.9 \%)$, and $51(21.6 \%)$ were frequently identified as co-infections, and HPV 16-58 and HPV 33-35 were the two most common combinations of HPV types.

\section{Discussion}

Our aim in this study was to investigate the distribution of HPV types in Korean women and evaluate the association between individual HPV types and the risk of precancerous lesions and cervical cancer. We also evaluated the potential effects of current HPV vaccines.

In HPV-positive patients, the most frequent HPV type was HPV 16, followed by HPV 58 and 68. In patients with HSIL+, HPV 16, 58, and 52 were frequently identified, with HPV 16 as the most prevalent type. Although previous studies in Korean populations have reported inconsistent results regarding the HPV type-specific distribution $^{17-19}$, those studies did agree that, compared with the worldwide prevalence ${ }^{20,21}$, HPV 58 is one of the most prevalent types in Korea, with HPV 16 and 18 being relatively less prevalent. This finding implies that the 9-valent vaccine, which covers five HR HPV types in addition HPV 16 and 18, would be beneficial to use in Korea.

In this study, the proportion of HR HPV types increased significantly from the NILM to the LSIL and HSIL+ cytology groups. However, not all HR HPV types were associated with HSIL + . The analysis of cervical cytology revealed that HPV 16, 18, 31, 33, 35, 51, 52, and 58 were significantly associated with HSIL + . Of those, HPV 16, $18,31,33$, and 58 were also associated with HSIL+ in histologically confirmed cases. Although HPV 59 showed a significant association with HSIL+ in the histologic evaluations, only six HPV 59 positive samples were included in the histologic analysis, so that the result should be interpreted with caution. Previous studies traced the disease progression of HPV-positive NILM or LSIL cytology patients and found that HPV 16, 18, 31, 33, 35, 52, and 58 were associated with the risk for HSIL $+{ }^{22-25}$, which is consistent with our findings. Because HR HPV genotypes confer significantly different prognostic information, it might be helpful to develop guidelines for specific HPV types to effectively manage patients.

HPV 16, 18, 31, 33, and 58, which all had high carcinogenic potential in both the cytology and histology results, are covered by the 9-valent vaccine. When the risk for HSIL+ was evaluated in the HR HPV subgroups according to vaccine coverage, the HPV types (HPV 31/33/45/52/58) covered by the 9 -valent vaccine were associated with twice the risk for HSIL + than the HR HPV types (HPV 35/39/51/56/59/66/68) not covered by the vaccine. Thus, the 9 -valent vaccine could be effective at preventing cervical cancer in the Korean population. In Korea, the 2-valent and 4-valent vaccines are currently included in the national immunization program as a 2-dose schedule for 12-year old girls. The 9-valent vaccine was approved by the Ministry of Food and Drug Safety in January 2016 and can be given as a non-national immunization program vaccine. Huh et al. reported that the 9-valent vaccine showed efficacy against HPV 31, 33, 45, 52, and 58 and similar efficacy toward HPV 6, 11, 16, and 18 as the 4 -valent vaccine ${ }^{26}$. In addition, 2 -doses of the 9 -valent vaccine showed non-inferior immunogenicity to the standard 3-doses in 10-14-year-old females ${ }^{27}$. Although factors such as cost should certainly be considered 
when determining a national immunization program, implementation of the 9 -valent vaccine might produce a substantial decrease in cervical cancer in Korea.

Among HPV-positive patients, the median age was youngest in the HSIL+ group, gradually increasing in the LSIL and NILM cytology groups. The prevalence of HSIL+ peaked in the 35-44 years group and decreased with age. These findings are in line with other recent studies in Korea ${ }^{18,28,29}$. Because women start being infected with HR HPV when they become sexually active, and HR HPV can cause HSIL in approximately 7 years, HSIL most commonly happens to women in their early $30 \mathrm{~s}^{30}$. In addition, the age-specific prevalence of CIN is attributable to the coverage of cervical cancer screening ${ }^{31,32}$. Because cervical cancer screening is registered as a national cancer screening program in Korea, the screened population could receive timely therapeutic intervention, allowing the prevalence of HSIL + to decrease in the older age groups.

This study has some limitations. First, it is a retrospective cross-sectional study. In addition, the HPV genotyping was performed with two different methods-the HPV 9G DNA Chip Test and the GeneFinder in the first and second halves of the study period, respectively. Although both methods have been validated ${ }^{33-35}$, no direct comparison of these two tests has been performed, so there could be discordance between them. Instead, we compared the results of the study periods covered by the different HPV genotyping methods. Of the total 28,834 cytology/HPV co-tested women, 13,697 patients (47.5\%) were tested using the DNA chip and 15,137 patients (52.5\%) were tested using the GeneFinder. The prevalence of HPV was $17.5 \%$ in the first period and slightly but not significantly increased to $18.3 \%$ in the second period. The prevalence of HSIL+ was $6.6 \%$ in the first period and $5.6 \%$ in the second period, while HPV 16 was identified in $1.8 \%$ and $1.5 \%$ in the first and second periods, respectively. Although the prevalence of HSIL + and individual HPV types did differ in the two periods, the genotyping tests were performed in different populations, making it difficult to identify any significant differences between the two methods. Nonetheless, this large-scale study identified the distribution of individual HPV types in Korean women and used risk stratification to assess the carcinogenic potential of specific HPV types. In addition, we found a high correlation between cytology and histology diagnoses, indicating that our results are reliable. This study thus provides guidance for improving the HPV-related national immunization program and management guidelines for individual HPV types.

\section{Methods}

Ethical statement. All specimens and related data were obtained with approval from the Institutional Review Board of Samsung Medical Center (SMC 2018-07-104) with a waiver of informed consent in accordance with HHS regulation 45CFR46.116(f). All methods were carried out according to the ethical standards of the institutional and national research committees and the Declaration of Helsinki.

HPV genotyping. HPV genotyping was performed with an HPV 9G DNA chip (Biometrix Technology Inc., Chuncheon, South Korea) or a GeneFinder HPV Liquid Bead MicroArray Genotype kit (GeneFinder; Infopia Inc., Anyang, South Korea). The HPV 9G DNA chip test was used from October 2014 to December 2015, and the GeneFinder was used from January 2016 to March 2017.

The HPV 9G DNA chip detects HPV genotypes via microarray, and the test was performed according to the manufacturer's protocol ${ }^{34}$. Briefly, HPV genomic DNA was extracted from cervicovaginal cytology samples and amplified via polymerase chain reaction (PCR). The PCR product was mixed with hybridization solution and loaded on the HPV 9G DNA chip. The DNA chip was then hybridized and washed at room temperature $\left(25^{\circ} \mathrm{C}\right)$. The final product was automatically scanned and analyzed.

The GeneFinder is a microsphere bead-based test that is highly specific and sensitive for detecting HPV genotypes $^{36}$. After HPV genomic DNA was extracted, the specimen was mixed with HPV type-specific polystyrene microspheres dyed with specific intensities of fluorophores. After hybridization and PCR amplification, the microspheres were streamed using Luminex technology (Luminex, Austin, TX). HPV genotypes were detected by a Luminex analyzer based on a flow cytometry method.

The HPV 9G DNA chip detects 19 HPV types (HPV 6, 11, 16, 18, 31, 33, 34, 35, 39, 40, 42, 45, 51, 52, 56, 58, 59,66 , and 68) and screens for 19 other types (HPV 3, 10, 26, 27, 32, 43, 44, 53, 54, 55, 57, 61, 62, 67, 69, 70, 71, 73, and 74) without specific typing. The GeneFinder detects 32 HPV types (HPV 6, 11, 16, 18, 26, 31, 32, 33, 34, 35, $39,40,42,43,44,45,51,52,53,54,55,56,58,59,62,66,68,69,70,73,81$, and 83 ). We analyzed the 19 HPV types (HPV 6, 11, 16, 18, 31, 33, 34, 35, 39, 40, 42, 45, 51, 52, 56, 58, 59, 66, and 68) detected by both tests. Among them, 14 HPV types (HPV 16, 18, 31, 33, 35, 39, 45, 51, 52, 56, 58, 59, 66, and 68), including IARC Groups 1 and $2 \mathrm{~A}^{11}$, are regarded as HR HPV, whereas HPV $6,11,34,40$, and 42 are regarded as LR HPV. If patients had an HPV type identified by one method but not the other, the HPV type was categorized as "other."

Statistical analysis. Patient ages were stratified into five subgroups: $\leq 34$ years, $35-44$ years, $45-54$ years, $55-64$ years, and $\geq 65$ years. A $\chi^{2}$ test or Fisher's exact test was used to evaluate the statistical significance of differences in categorical variables among groups. Post-hoc test was performed using Tukey's Honestly Significant Difference test, and p-values from multiple comparisons were corrected by the Bonferroni method. Age was additionally analyzed as a continuous variable using the Kruskal-Wallis test followed by Dunn's post-hoc test. To assess the association of HPV subtypes with HSIL+, we performed logistic regressions and calculated the OR and 95\% CI. For HPV types, the OR was defined as the ratio between the odds of being in the HSIL+ group in the presence of an individual HPV type and the odds of being in the HSIL+ group in the absence of an individual HPV type. The results were visualized by forest plot using the SGPLOT procedure. For all analyses, $\mathrm{p}<0.05$ was considered statistically significant, and all analyses were two-sided. Data were analyzed using SAS version 9.4 (SAS Institute Inc., Cary, NC). 


\section{Data Availability}

Most data are provided in the manuscript and Supplementary Information. Other data will be available from the corresponding author upon reasonable request.

\section{References}

1. Walboomers, J. M. et al. Human papillomavirus is a necessary cause of invasive cervical cancer worldwide. J. Pathol. 189, 12-9 (1999).

2. Kocjan, B. J., Bzhalava, D., Forslund, O., Dillner, J. \& Poljak, M. Molecular methods for identification and characterization of novel papillomaviruses. Clin. Microbiol. Infect. 21, 808-816 (2015).

3. Muñoz, N. et al. Epidemiologic Classification of Human Papillomavirus Types Associated with Cervical Cancer. N. Engl. J. Med. 348, 518-527 (2003).

4. Bouvard, V. et al. A review of human carcinogens-Part B: biological agents. Lancet. Oncol. 10, 321-2 (2009).

5. Clifford, G. M., Smith, J. S., Plummer, M., Muñoz, N. \& Franceschi, S. Human papillomavirus types in invasive cervical cancer worldwide: A meta-analysis. Br. J. Cancer 88, 63-69 (2003).

6. Bosch, F. X. et al. Prevalence of human papillomavirus in cervical cancer: a worldwide perspective. International biological study on cervical cancer (IBSCC) Study Group. J. Natl. Cancer Inst. 87, 796-802 (1995).

7. de Sanjose, S. et al. Human papillomavirus genotype attribution in invasive cervical cancer: a retrospective cross-sectional worldwide study. Lancet Oncol. 11, 1048-1056 (2010).

8. Clifford, G. M. et al. Worldwide distribution of human papillomavirus types in cytologically normal women in the International Agency for Research on Cancer HPV prevalence surveys: A pooled analysis. Lancet 366, 991-998 (2005).

9. Sanjosé, S., Díaz, M., Castellsagué, X., Clifford, G. \& Bruni, L. Worldwide prevalence and genotype distribution of cervical HPV in women with normal cytology. Lancet Infect 7, 453-459 (2007).

10. Parkin, D. M., Louie, K. S. \& Clifford, G. Burden and trends of type-specific human papillomavirus infections and related diseases in the Asia Pacific region. Vaccine 26, M1-M16 (2008).

11. IARC Working Group on the Evaluation of Carcinogenic Risks to Humans. \& International Agency for Research on Cancer. A review of human carcinogens. Biological agents. (International Agency for Research on Cancer, 2012).

12. Powell, N. G. et al. The risk of cervical cancer associated with specific types of human papillomavirus: A case-control study in a UK population. Int. J. Cancer 128, 1676-1682 (2011).

13. Schiffman, M. et al. A population-based prospective study of carcinogenic human papillomavirus variant lineages, viral persistence, and cervical neoplasia. Cancer Res. 70, 3159-3169 (2010).

14. Hildesheim, A. et al. Human Papillomavirus Type 16 Variants and Risk of Cervical Cancer. JNCI J. Natl. Cancer Inst. 93, 315-318 (2001).

15. Solomon, D. et al. The 2001 Bethesda System: terminology for reporting results of cervical cytology. JAMA 287, 2114-9 (2002).

16. Lee, J. K. et al. Practice guidelines for the early detection of cervical cancer in Korea: Korean society of Gynecologic Oncology and the Korean society for Cytopathology 2012 edition. J. Gynecol. Oncol. 24, 186-195 (2013).

17. Ouh, Y. et al. Prevalence of human papillomavirus genotypes and precancerous cervical lesions in a screening population in the Republic of Korea, 2014-2016. 29, 1-10 (2018).

18. Lee, E. H. \& Um, T. H. Prevalence and Distribution of Human Papillomavirus Infection in Korean Women as Determined by Restriction Fragment Mass Polymorphism Assay. 1091-1097 (2012).

19. Kim, M.-J., Kim, J. J. \& Kim, S. Type-specific prevalence of high-risk human papillomavirus by cervical cytology and age: Data from the health check-ups of 7,014 Korean women. Obstet. Gynecol. Sci. 56, 110-20 (2013).

20. de Sanjosé, S. et al. Worldwide prevalence and genotype distribution of cervical human papillomavirus DNA in women with normal cytology: a meta-analysis. Lancet Infect. Dis. 7, 453-459 (2007).

21. Bruni, L. et al. Cervical Human Papillomavirus Prevalence in 5 Continents: Meta-Analysis of 1 Million Women with Normal Cytological Findings. J. Infect. Dis. 202, 1789-1799 (2010).

22. Schiffman, M. et al. Gynecologic Oncology A study of HPV typing for the management of HPV-positive ASC-US cervical cytologic results. Gynecol. Oncol. 138, 573-578 (2015).

23. Lie, A. K. et al. HPV genotype profile in a Norwegian cohort with ASC-US and LSIL cytology with three year cumulative risk of high grade cervical neoplasia. Gynecol. Oncol. 148, 111-117 (2018).

24. Kang, W. D., Ju, U. C. \& Kim, S. M. Is human papillomavirus genotype important in predicting disease progression in women with biopsy-proven negative or CIN1 of atypical squamous cell of undetermined significance (ASC-US) cytology? Gynecol. Oncol. 148, 305-310 (2017).

25. Monsonego, J. et al. Prevalence of high-risk human papilloma virus genotypes and associated risk of cervical precancerous lesions in a large U.S. screening population: Data from the ATHENA trial. Gynecol. Oncol. 137, 47-54 (2015).

26. Huh, W. K. et al. Final efficacy, immunogenicity, and safety analyses of a nine-valent human papillomavirus vaccine in women aged 16-26 years: a randomised, double-blind trial. Lancet 390, 2143-2159 (2017).

27. Iversen, O.-E. et al. Immunogenicity of the 9-Valent HPV Vaccine Using 2-Dose Regimens in Girls and Boys vs a 3-Dose Regimen in Women. JAMA 316, 2411 (2016).

28. Shim, S. H. et al. Nationwide cervical cancer screening in Korea: Data from the national health insurance service cancer screening program and national cancer screening program, 2009-2014. J. Gynecol. Oncol. 28, 1-12 (2017).

29. Chang, H. K., Seo, S. S., Myong, J. P., Yu, Y. L. \& Byun, S. W. Incidence and costs of cervical intraepithelial neoplasia in the Korean population. J. Gynecol. Oncol. 30, e37 (2019).

30. Schlecht, N. F. et al. Human Papillomavirus Infection and Time to Progression and Regression of Cervical Intraepithelial. Neoplasia. CancerSpectrum Knowl. Environ. 95, 1336-1343 (2003).

31. Ting, J., Kruzikas, D. T. \& Smith, J. S. A Global Review of Age-Specific and Overall Prevalence of Cervical Lesions. Int. J. Gynecol. Cancer 20, 1244-1249 (2010).

32. Qiao, Y. L. et al. Prevalence of human papillomavirus and cervical intraepithelial neoplasia in China: A pooled analysis of 17 population-based studies. Int. J. Cancer 131, 2929-2938 (2012).

33. Jun, S. Y. et al. Comparison of the cobas $4800 \mathrm{HPV}$ and HPV 9G DNA chip tests for detection of high-risk human papillomavirus in cervical specimens of women with consecutive positive HPV tests but negative pap smears. PLoS One 10, 1-11 (2015).

34. An, H. et al. HPV 9G DNA chip: 100\% clinical sensitivity and specificity. J. Clin. Microbiol. 50, 562-568 (2012).

35. Ko, K., Kwon, M. J., Lee, E. H., Woo, H. Y. \& Park, H. Comparison of GeneFinder human papillomavirus (HPV) Liquid Beads Microarray PCR Kit and Hybrid Capture 2 Assay for Detection of HPV Infection. J. Clin. Lab. Anal. 31 (2017).

36. Schmitt, M. et al. Abundance of multiple high-risk human papillomavirus (HPV) infections found in cervical cells analyzed by use of an ultrasensitive HPV genotyping assay. J. Clin. Microbiol. 48, 143-149 (2010). 


\section{Author Contributions}

E.P.: Conceptualization, data curation, formal analysis, investigation, writing-original draft, and writing-review and editing. J.-Y.K.: Conceptualization, data curation and editing. S.C.: Data curation. D.S.K.: Methodology and resources. Y.L.O.: Conceptualization, investigation, project administration, writing-review and editing, and supervision.

\section{Additional Information}

Supplementary information accompanies this paper at https://doi.org/10.1038/s41598-019-49060-w.

Competing Interests: The authors declare no competing interests.

Publisher's note: Springer Nature remains neutral with regard to jurisdictional claims in published maps and institutional affiliations.

(c) (i) Open Access This article is licensed under a Creative Commons Attribution 4.0 International License, which permits use, sharing, adaptation, distribution and reproduction in any medium or format, as long as you give appropriate credit to the original author(s) and the source, provide a link to the Creative Commons license, and indicate if changes were made. The images or other third party material in this article are included in the article's Creative Commons license, unless indicated otherwise in a credit line to the material. If material is not included in the article's Creative Commons license and your intended use is not permitted by statutory regulation or exceeds the permitted use, you will need to obtain permission directly from the copyright holder. To view a copy of this license, visit http://creativecommons.org/licenses/by/4.0/.

(C) The Author(s) 2019 